\title{
Deterministic and probabilistic deep learning models for inverse design of broadband acoustic cloak
}

\author{
Waqas W. Ahmed $\odot$, Mohamed Farhat, Xiangliang Zhang, ${ }^{*}$ and Ying $\mathrm{Wu}^{\dagger}$ \\ Division of Computer, Electrical and Mathematical Science and Engineering, \\ King Abdullah University of Science and Technology (KAUST), Thuwal, 23955-6900, Saudi Arabia
}

(Received 23 November 2020; revised 19 January 2021; accepted 22 January 2021; published 12 February 2021)

\begin{abstract}
Concealing an object from incoming waves (light and/or sound) remained science fiction for a long time due to the absence of wave-shielding materials in nature. Yet, the invention of artificial materials and new physical principles for optical and sound wave manipulation translated this abstract concept into reality by making an object optically and acoustically "invisible." Here, we present the notion of a machine learning driven acoustic cloak and demonstrate an example of such a cloak with a multilayered core-shell configuration. We develop deterministic and probabilistic deep learning models based on autoencoderlike neural network structure to retrieve the structural and material properties of the cloaking shell surrounding the object that suppresses scattering of sound in a broad spectral range, as if it was not there. The probabilistic model enhances the generalization ability of design procedure and uncovers the sensitivity of the cloak's parameters on the spectral response for practical implementation. This proposal opens up avenues to expedite the design of intelligent cloaking devices for tailored spectral response and offers a feasible solution for inverse scattering problems.
\end{abstract}

DOI: 10.1103/PhysRevResearch.3.013142

\section{INTRODUCTION}

Cloaking or invisibility, physically related to the cancellation of the natural scattering signature of an object, has attracted enormous attention. In order to render an object invisible to electromagnetic and/or acoustic wave, it is essential to tailor the interaction of waves with the object such that the wavefronts in the surrounding medium remain undisturbed, regardless of the presence of an object. One promising route to achieve invisibility is via transformation optics, relying on artificially structured materials [1] that can mold the flow of waves around the concealed object with specific design of its constitutive parameters. However, such transformation-based cloaks [2-4] have the fundamental limitation of narrowband operation, due to the strong dispersion, inherent to the resonance-based meta-atoms, and the undesired material loss; they are thus challenging due to the difficulty of creating bulky material compositions, with both anisotropy and inhomogeneity $[4,5]$. In such scenarios, other invisibility schemes based on scattering cancellation technique [6,7], patterned metasurfaces [8,9], and complex modulated potentials [10,11] have also been suggested for different kinds of waves ranging from microwave $[4,12,13]$, acoustic [14-16], and elastic [17] to heat waves [18].

\footnotetext{
*xiangliang.zhang@ kaust.edu.sa

$\dagger$ ying.wu@kaust.edu.sa

Published by the American Physical Society under the terms of the Creative Commons Attribution 4.0 International license. Further distribution of this work must maintain attribution to the author(s) and the published article's title, journal citation, and DOI.
}

In the past decade, acoustic cloaks via scattering cancellation $[19,20]$ have become a topic of interest due to their robust designs, operating spectral range and ease of fabrication. In such schemes, isotropic layers of specific thickness, mass density, and bulk modulus can be carefully tailored to cancel the first few scattering orders, which significantly reduce the scattering cross section of the system, to make the object nearly undetectable at a particular frequency. Consequently, the scattering cancellation approach generally employs acoustic metamaterials to realize on-demand cloaking devices. However, practical applications often desire more flexibility in the operating frequency band and require designing materials with positive physical properties (density and bulk modulus). Yet, the design of cloaking shells operating over broad frequency ranges with realistic material parameters remains challenging. For instance, the broadband cloaking operation requires some additional layers in the core-shell configuration to cancel the higher scattering orders and, as a consequence, the design complexity grows and thus makes it extremely challenging to tune the geometry and material properties with conventional optimization techniques [21,22]. In addition, conventional optimization methods relying on the evolutionary algorithms suffer from a local minima problem and require prior knowledge on the selection of objective function for accurate design. To mitigate such issues, datadriven approaches based on machine learning have provided a promising platform where artificial neural networks are trained to intelligently learn the intrinsic relation between various structural parameters and their spectral responses, and significantly reduce the overall computational time by predicting the solution immediately after the training phase $[23,24]$.

The rapid development in machine learning technology enables people to efficiently solve numerous physical 
(a)
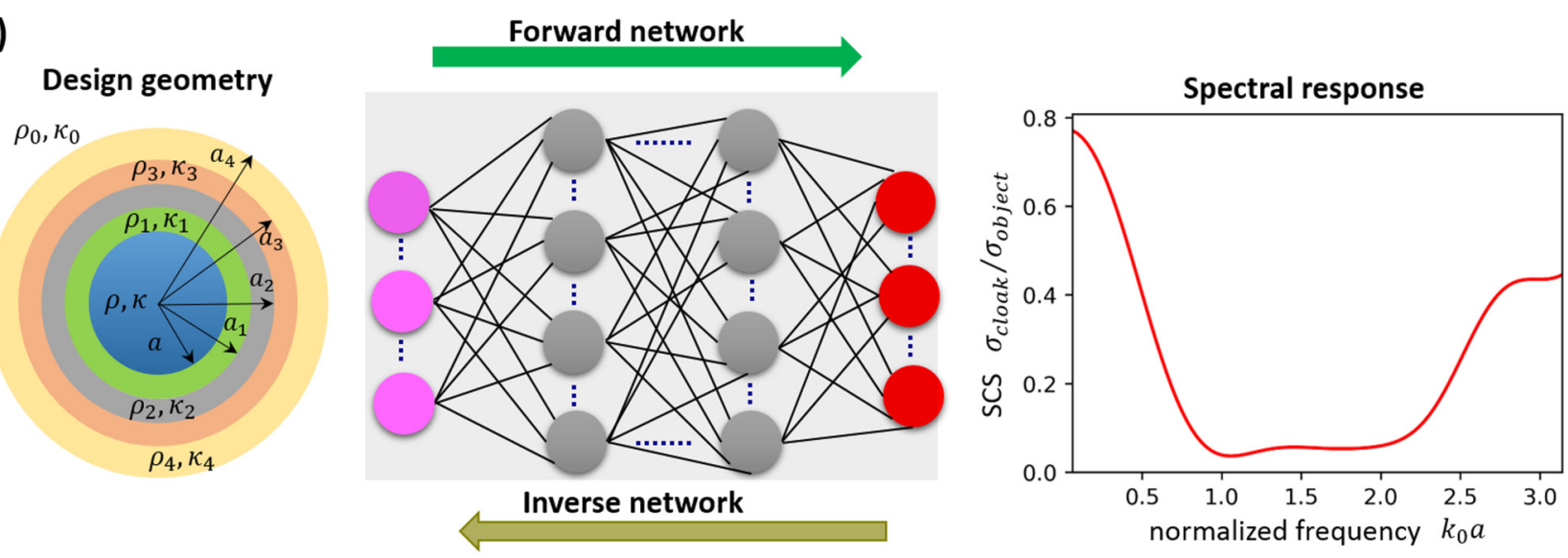

(b)
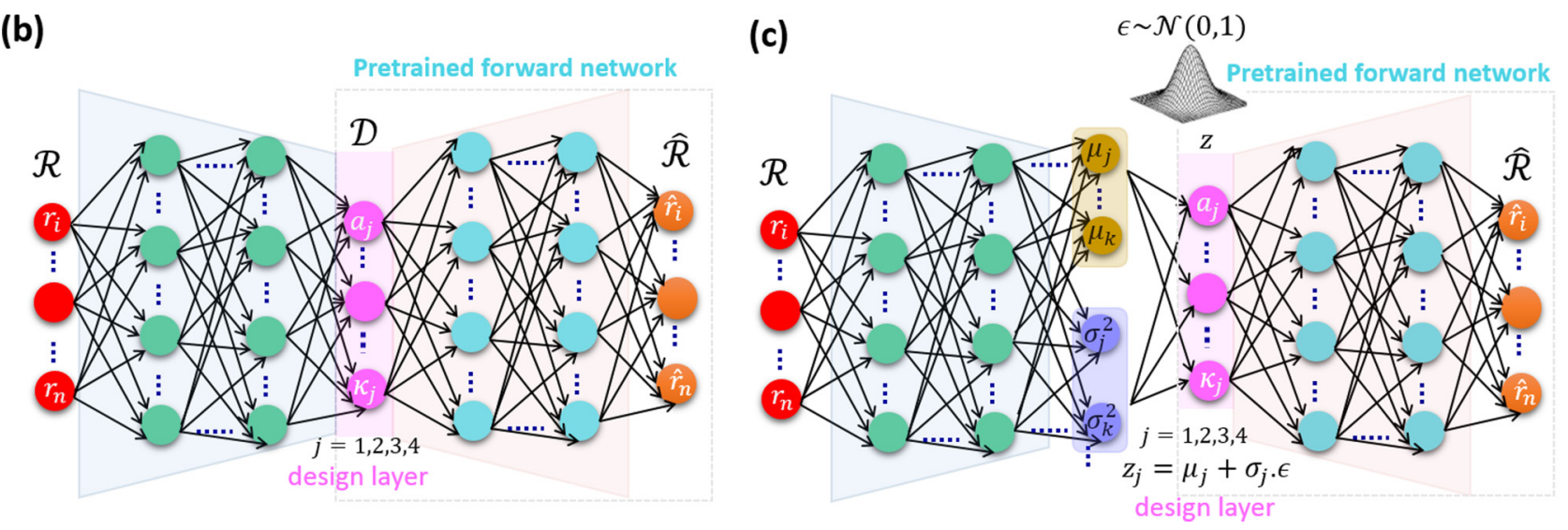

FIG. 1. Framework of the deep learning network for inverse design of the acoustic cloak. (a) Schematic illustration of the core-shell acoustic cloak and its spectral response (ratio between total scattering cross-section spectra of the cloak $\sigma_{\text {cloak }}$ and that of the object, i.e., $\sigma_{\text {object }}$ ) where the neural network learns the relation from $\mathcal{D}$ (design parameters) to $\mathcal{R}$ (spectral response) and from $\mathcal{R}$ to $\mathcal{D}$ for forward and inverse design, respectively. (b), (c) Proposed deep learning models for inverse design of the cloak. (b) Deterministic model where the pretrained forward network acts as a decoder to predict the spectral response. (c) Probabilistic model where the design space is transformed into the latent space $z$, with a standard Gaussian distribution. The physical design parameters are sampled from that distribution in the form of latent variables to generate the desired spectral response.

problems including quantum physical problems [25], modulation instability in optical fibers [26], and pattern recognition of photonic modes [27], and streamline the inverse design process of protected edge states [28], metasurfaces [29-31], and complex structures for different applications [32-44]. The inverse design process allows for fast and accurate prediction of the design parameters (structure and material properties) with complex architectures such as deep neural networks (DNNs) [29,32], convolutional neural networks (CNNs) [34], recurrent neural network (RNNs) [40], and generative adversarial networks (GANs) [29]. Despite such significant advancement in this area, the reported studies to date mostly emphasize solving inverse electromagnetic problems in a deterministic fashion, while robust deep learning models for inverse acoustic scattering problems are yet to be developed. Here, we propose deep learning models as a practical tool to design broadband acoustic cloaks using a core-shell configuration. The proposed model utilizes fully connected DNNs to capture and generalize the nonlinear intricate relation between the design parameters and the spectral response for the forward and the inverse problem [see Fig. 1(a)]. The implementation of the forward problem is straightforward, and consists in training the neural network that maps the design parameters directly to the spectral response, but the inverse design is intrinsically challenging due to the nonuniqueness of the solution and inherent convergence problems [33,34]. To address these issues, we design an autoencoderlike structure consisting of two DNNs where the pretrained forward network is cascaded behind the inverse neural network that maps the spectral response to the design parameters in either a deterministic [see Fig. 1(b)] or a probabilistic manner [see Fig. 1(c)]. The deterministic inverse design network provides only one set of design parameters for a given spectral response. However, a practical implementation generally demands more flexibility and diversity in the design due to external perturbation. Hence, we introduce a model to provide probabilistic distributions of the design parameters, which flexibly generates the desired spectral response [see Fig. 1(c)]. The probabilistic design with parameter distributions is more advanced than the deterministic design with fixed parameters on the basis of twofold benefits (i) the capability to generate a variety of design parameters for one desired spectral response and (ii) the ability to uncover the sensitivity of the design parameters on the cloaking effect. We find that the bulk modulus (physical parameter) is less sensitive to external perturbation than the thickness (geometric parameter) of the layers in designing the 
acoustic cloaks. To verify the predicted cloaking profile, we also perform full-wave simulations based on the finite element method (FEM) and obtain excellent cloaking effect. Notably, this framework allows us to design broadband cloaks using realistic materials that can be readily achievable for stealth technologies.

\section{DEEP LEARNING MODEL}

We consider a two-dimensional acoustic cloaking problem. Our purpose is to make a cylinder, indicated by the blue core in Fig. 1(a), acoustically invisible. To achieve this goal, we consider a four concentric layered shell configuration where each layer is made of different material with different outer radius as illustrated in Fig. 1(a). The properties of the cylindrical scatterer are described by its radius $a$, mass density $\rho$, and bulk modulus $\kappa$, while the $m$ th cloak's layer is characterized by the outer radius $a_{m}$ and material properties $\left(\rho_{m}, \kappa_{m}\right)$, where $m=1,2,3,4$ is the layer index. In order to analyze the scattering response of this system, we make use of the transfer matrix method (TMM) and compute the total scattering cross-section (SCS) spectra (see the Supplemental Material (SM) [45] for details). To quantify the cloak's performance, we define the ratio of the SCS spectra of the cloaked object $\sigma_{\text {cloak }}$ and the bare object $\sigma_{\text {object }}$, i.e., $\sigma_{\text {cloak }} / \sigma_{\text {object }}$ (or normalized SCS). This ratio reveals how well the object becomes acoustically invisible with the presence of the designed cloak. The ideal cloaking behavior is achieved by optimizing the design parameters to yield a near-zero SCS at the operation frequency. Without loss of generality, we set the scatterer's parameters as $a=1 \mathrm{~m}, \rho=\rho_{0}$, and $\kappa=1.5 \kappa_{0}$, with $\rho_{0}$ and $\kappa_{0}$ being the mass density and the bulk modulus of the host medium, respectively. We use the TMM to generate the training data samples, where we determine the SCS spectra for random design space, $\mathcal{D}=\left[a_{1}, a_{2}, a_{3}, a_{4}, \kappa_{1}, \kappa_{2}, \kappa_{3}, \kappa_{4}\right]$, with $a_{m}$ and $\kappa_{m}$ the radius and bulk modulus of the $m$ th layer, respectively, while keeping the density fixed in each layer. Each training example is represented by eight design parameters (four radii and four bulk moduli) and 100 discrete points of SCS spectrum $\mathcal{R}=\left[r_{1}, r_{2}, r_{3}, r_{4} \cdots r_{100}\right]$ covering the normalized frequency range $0 \leqslant k_{0} a \leqslant \pi$, with $k_{0}=2 \pi / \lambda$ the acoustic wave number. We design the forward neural network to map the design $\mathcal{D}$ to the spectrum $\mathcal{R}$, and the inverse model to map the spectrum $\mathcal{R}$ to the design $\mathcal{D}$. Both networks are trained by optimizing the neural network weights. For our analysis, we generate 68000 data samples for random design parameters, which are split into three distinct groups: 60000 data samples for training, 4000 data samples for validation, and 4000 data samples for final testing. The training data are used to train the network by optimizing the neural network weights, while the validation data set serves for checking and avoiding the overfitting issue, and the testing data set examines the prediction accuracy of the network.

\section{A. Forward-modeling network}

We first design the forward-modeling network to accurately predict the frequency-dependent SCS for given design parameters. The forward model builds a fully connected network between the design space $\mathcal{D}$ as the input layer and SCS
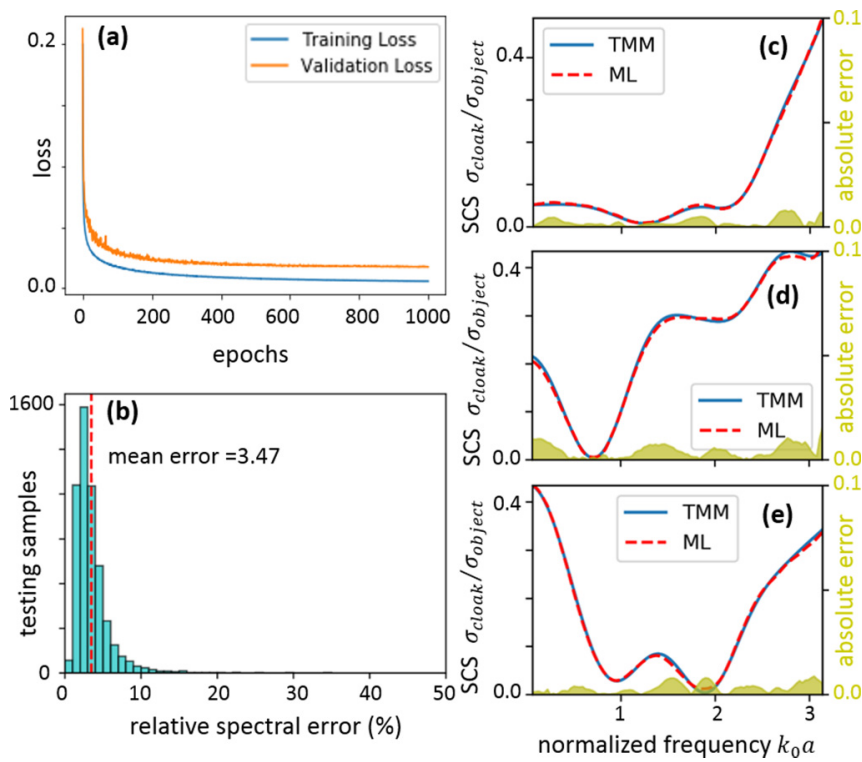

FIG. 2. Forward network learning of acoustic cloak. (a) Learning curves for training and validation data sets as a function of training epochs. (b) Histogram of relative spectral error for testing samples. The red vertical dashed line shows the mean spectral error. (c)-(e) Comparison of the spectral response for three representative examples obtained by machine learning model and TMM. The shaded area shows the absolute error between the predicted and target response, which indicates that the machine learning results are in perfect agreement with those of TMM. The design parameters are provided in the SM [45].

spectra $\mathcal{R}$ as the output layer, as shown in Fig. 1(a). We normalize the data before training, to expedite the convergence of the network. In the training process, the training data are fed into the network and the weights are continuously optimized to minimize the loss function defined as $\mathcal{L}=\frac{1}{N} \sum_{k}\left|r_{k}-\hat{r}_{k}\right|$ where $r_{k}$ and $\hat{r}_{k}$ are the ground truth of the spectral response and the response predicted by the neural network, respectively. Note that we used the mean absolute error as a cost function for the training purpose due to the presence of outliers in the data. The architecture of the forward network is optimized to have four fully connected layers with each layer having 500-500-500-300 nodes, respectively. The remaining hyperparameters (batch size, learning rate, activation function, etc.) are judiciously selected to minimize the validation loss (see the SM [45]). The learning curves for the training and validation data as a function of epoch are shown in Fig. 2(a). Both the training and validation errors drop as the training goes on, until it converges after 400 epochs, implying the completion of the training phase. Due to the unique one-to-one mapping between design space and response in the forward problem, the training process is straightforward to converge. To check the prediction accuracy of the trained model, we evaluate it on a group of 4000 data samples that are not seen in the training process. We predict the spectral response of the trained network for testing data and compare the results with those obtained by the TMM. We report the relative absolute spectral error on the testing data sets as $e=\sum_{k}\left|r_{k}-\hat{r}_{k}\right| / r_{k}$ where $r_{k}$ is the discretized value for the target spectral response and $\hat{r}_{k}$ is the corresponding 
predicted spectral response. The results for relative spectral error are plotted in Fig. 2(b) with a mean error of $3.47 \%$ for testing data, which proves the high prediction accuracy (over $96 \%$ ) of the network. Figures 2(c)-2(e) depict the spectral response of three representative cases from the testing data that clearly indicate the predicted results perfectly match those of the TMM.

\section{B. Deterministic inverse-modeling network}

Typically, practical applications demand the cloaking effect at a particular frequency or over a broad frequency band, which requires realizable design parameters to produce the desired spectral response. Yet, there is no common tool available for the accurate inverse design of cloaking devices. The development of such tools significantly reduces the computational time for design optimization and accelerates the generation of the desired SCS spectra. To achieve this goal, we attempt to train the network inversely, which takes the spectral response as the input and the design parameters as the output. However, we are unable to train the network successfully in the inverse direction due to nonunique solutions in response to design mapping that leads to nonconvergence issues [(see the SM [45] for more details). To resolve this issue, we implement the autoencoderlike network where we cascade the inverse network to the independently trained (or pretrained) forward-modeling network, as shown in Fig. 1(b). The forward network is trained separately to substitute the TMM simulation and acts as a data generator in training. The pretrained forward network accelerates the inverse design process due to fast computation time ( $\sim$ millisecond $)$ as compared to TMM ( second) while maintaining good prediction accuracy for the designed spectral response. During the training process, the pretrained forward network has fixed weights and biases while the weights of the inverse network are updated iteratively to minimize the loss function to the output response of the designed structure predicted by the neural network. The designed structure refers to the intermediate layer $\mathcal{D}$ in the autoencoderlike network that predicts the eightdesign parameter for the desired spectral response. Figure 3(a) depicts the learning curves for the training and validation loss that decrease rapidly and converge after 1000 epochs of training. The inverse network architecture is designed to have five fully connected layers with each layer composed of 500-500-500-500-400 nodes, respectively. Details about the hyperparameters are discussed in the SM [45]. Again, we use 4000 testing data sets to examine the accuracy of the inverse design network. Figure 3(b) shows the histogram of relative spectral error, where the average spectral error across all predicted spectra is $4.63 \%$. The scattering response for representative cases (c-e) confirms the accuracy of the designed inverse network.

To verify the predicted cloaking effective in the broad spectral range, we perform full-wave numerical simulations and plot the results in Fig. 4, which depicts the real part of the pressure field distributions of the bare object and the cloaked object under the excitation of a plane wave at different frequencies. The results are obtained by the finite element method (COMSOL MULTIPHYSICS). It shows the incident waves are severely distorted in the case of the bare object, indicating
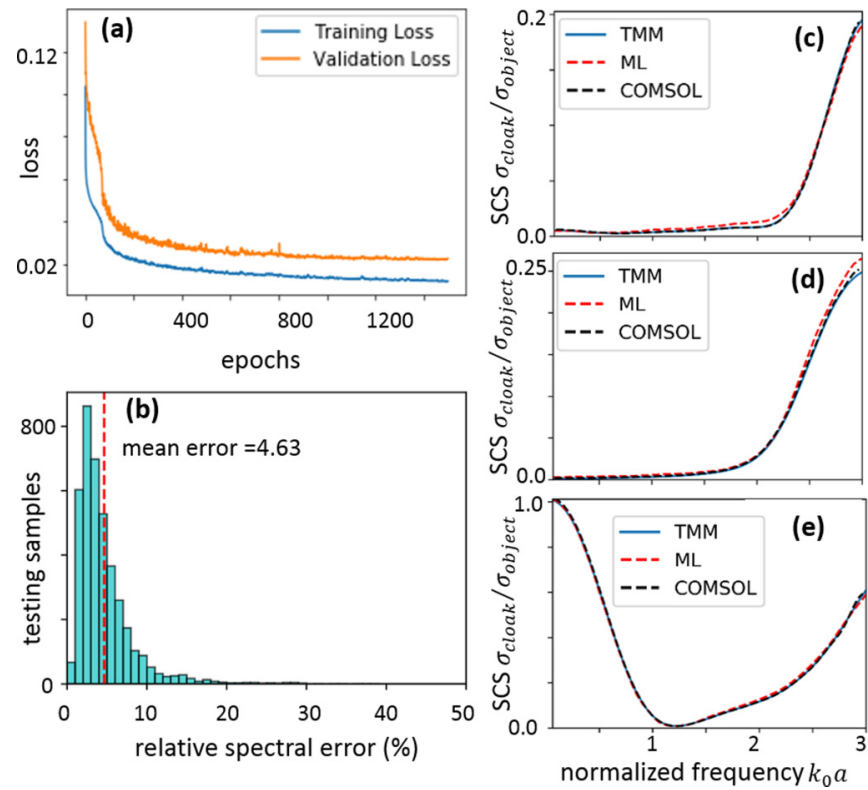

FIG. 3. Deterministic inverse design of acoustic cloak. (a) Learning curves for training and validation data sets as functions of the training epochs. (b) Histogram of the relative spectral error for the testing data samples. The red vertical dashed line shows the mean spectral error. (c)-(e) Comparison of the spectral response for three representative cases obtained with machine learning, TMM, and COMSOL. These results clearly show that machine learning accurately predicts the target response. The designed responses require positive mass density and bulk modulus values that are provided in the SM [45].

strong scattering behavior. However, the field distributions with the presence of the cloaked object are similar to those without any object, as if the cloaked object does not exist, validating the performance of the designed cloak, in a broad spectral range.

\section{Probabilistic inverse-modeling network}

In the deterministic inverse design, we choose one precise set of design parameters to generate the desired spectral response, yet the practical implementation demands diversity in design parameters due to the possible unavailability of actual materials and deviations from the original design parameters. In this scenario, it is essential to enhance the generalization and robustness of our network by introducing the probabilistic prediction. To achieve this goal, we propose the stochastic inverse design that uses the latent space concept [36] for the probabilistic representation of the physical design parameters. Our probabilistic inverse design network is basically a generative model which transforms the input spectral response into a mean vector $\mu$ and a variance vector $\sigma^{2}$ to approximate the distribution of the latent variables corresponding to the design space, and then the pretrained forward network, acting as a decoder, generates the same spectral response as the input by sampling latent variable vector $z$ from the Gaussian prior distribution illustrated in Fig. 1(c). Note that latent variables in such an architecture are strongly correlated with the physical parameters (i.e., thicknesses and bulk modulus of the layers), 
(a)
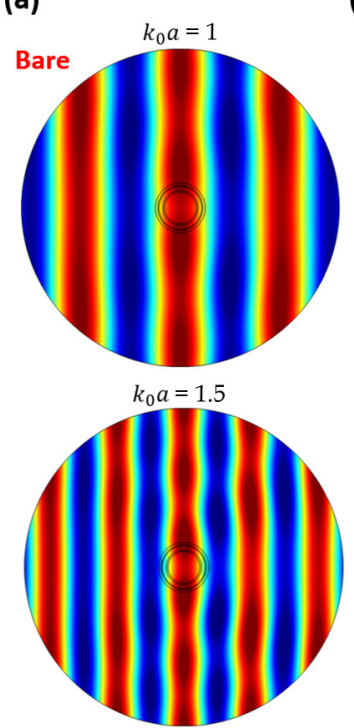

$k_{0} a=2$

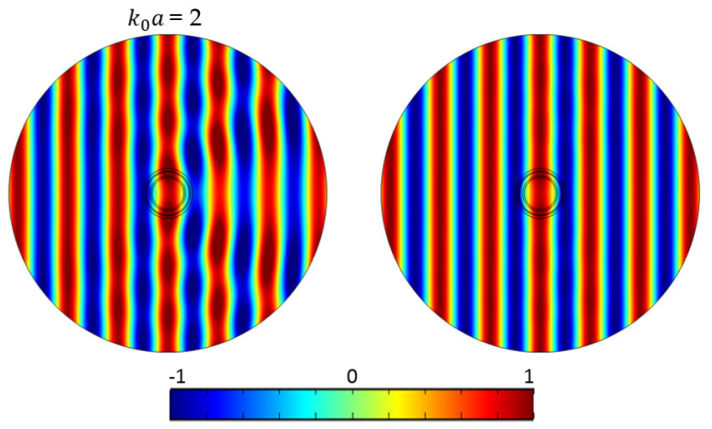

FIG. 4. Numerical simulations for designed broadband acoustic cloak. (a) Pressure field distribution produced by an incident plane wave for the bare object and (b) the cloaked object. The three different normalized frequencies $k_{0} a=1,1.5,2$ are picked from the spectral response shown in Fig. 2(c).

indicating that the network automatically learns the physical relation between the design and the spectral responses. The loss function for the probabilistic inverse network consists of mean absolute error for reconstruction $\mathcal{L}_{\mathrm{MAE}}$, a KullbackLeibler $(\mathrm{KL})$ divergence error $\mathcal{L}_{\mathrm{KL}}$, and a precision parameter, i.e., the inverse of the variance $\tau_{p}=1 / \sigma^{2}$. The KL divergence ensures the generated latent space distributions follow the assumed Gaussian distribution, $\mathcal{N}(0,1)$, and also provides the discrepancy between the predicted distributions and the standard normal distribution. The precision term is incorporated to avoid the zero-variance problem for the generated distributions of the latent space. The model is trained to minimize the following loss function:

$$
\mathcal{L}=\frac{1}{N} \sum_{k=1}^{N}\left(\mathcal{L}_{\mathrm{MAE}}^{(k)}+\alpha \mathcal{L}_{\mathrm{KL}}^{(k)}+\beta \tau_{p}^{(k)}\right),
$$

where $N$ is the total number of training samples, $\alpha$ is the weight of probabilistic learning, and $\beta$ is the regularization parameter. These hyperparameters can be tuned by cross validation during the training process. The detail of the derived cost function is provided in the SM [45].
Figure 5(a) shows the training and validation learning curves as a function of the training epoch, where the loss function for both training and testing data sets converges after 2500 epochs. The architecture of the stochastic inverse model is designed to have nine fully connected layers with each layer having 500-500-500-500-500-500-500-500-400 hidden neurons, respectively (see the details about the hyperparameters in the SM [45]). The designed network essentially learns the distribution of the design parameters conditioned on its spectral response. After successful training, we explore the generated distributions of the design space for the testing data. The standard deviation of the generated distribution indicates the range of design parameters that can generate the same spectral response. It also reveals the sensitivity of the design parameters on the generation of the spectral response.

Quantitatively, we can classify the spectral responses by finding the maximum standard deviation in the latent distributions of the design parameters for testing data sets. We observe that the distributions of the bulk modulus parameters exhibit larger standard deviations as compared to the thicknesses in the design space. For the analysis, we set criteria of minimum $\pm 2 \%$ and $\pm 4 \%$ standard deviation (from the mean value of the corresponding design parameter) in the distribution and categorize the predicted design parameters in testing samples based on the maximum standard deviation, as depicted in Fig. 5(b). We further sort the design space in testing data based on minimum $\pm 2 \%$ and $\pm 4 \%$ standard deviation (with respect to mean value) in different combinations of bulk modulus parameters as shown in Fig. 5(c). Such classifications identify the tolerance of the bulk moduli to generate the same spectral response. Figures $5(\mathrm{~d})-5(\mathrm{~g})$ show the spectral response of four representative cases with maximum standard deviation in the bulk modulus distributions. The randomly sampled bulk moduli in the corresponding distribution (indicated by dashed red and green curves) generate the same spectral responses, showing the excellent performance of our stochastic design (see more examples in the SM [45]).

\section{DISCUSSION AND CONCLUSION}

We use supervised and semisupervised learning algorithms based on the nonlinear regression technique to develop a deep learning model that maps the design space to the spectral response for the forward design and the spectral response to the design space in the inverse design of the acoustic cloak. The mapping functions are implemented by deep learning neural networks. To solve the inverse problem, we design the deterministic and probabilistic encoder-decoder like networks. The deterministic design encodes the spectral response into the design parameters, which are decoded with the pretrained forward model to generate the desired response. Yet, the probabilistic design models the statistical distribution of the design parameters. Such distributions allow the diversity and flexibility for fabrication and application of the designed cloak. The training of the probabilistic design process includes four basic steps: First, the spectral response is transformed into a distribution over the latent space. Second, a point from the latent space is randomly sampled from that distribution. Third, the sampled point is decoded using a pretrained forward 
(a)

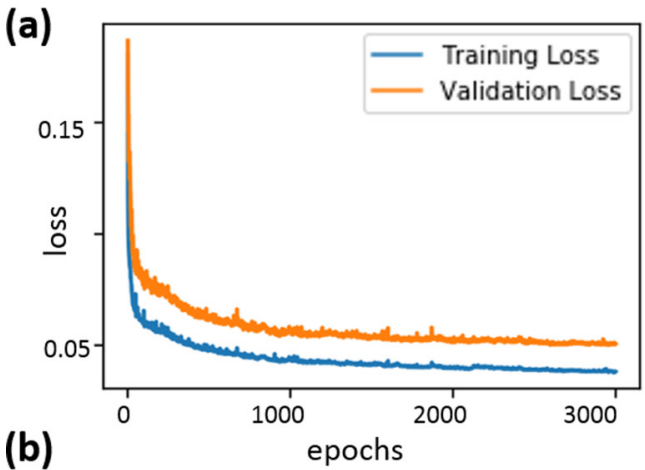

(b)

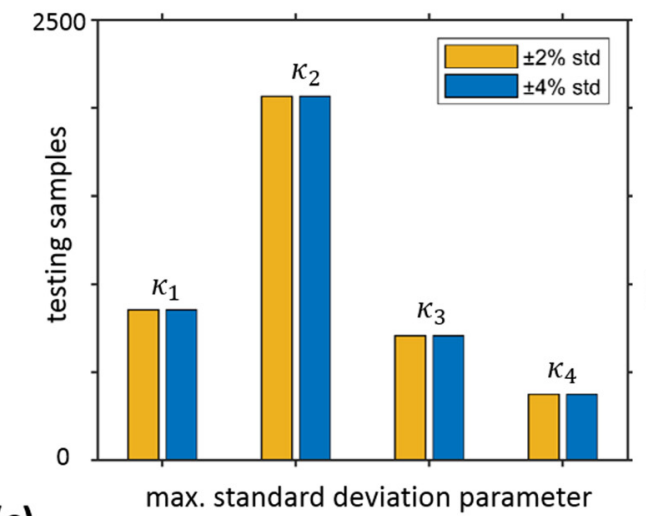

(c)

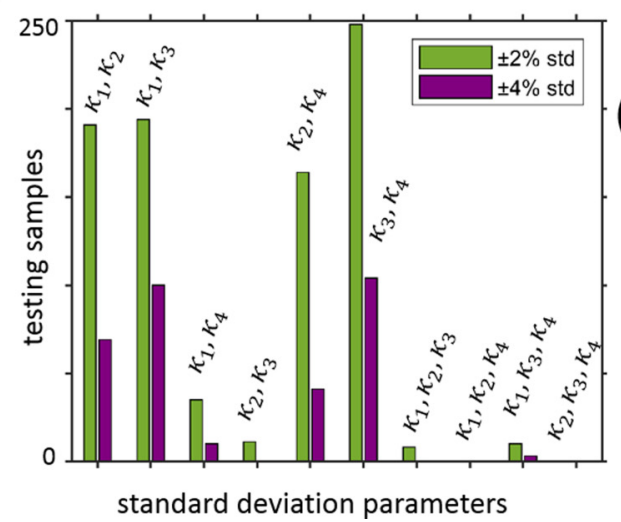

(d)

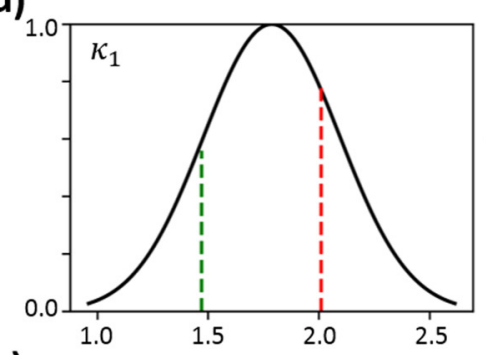

(e)
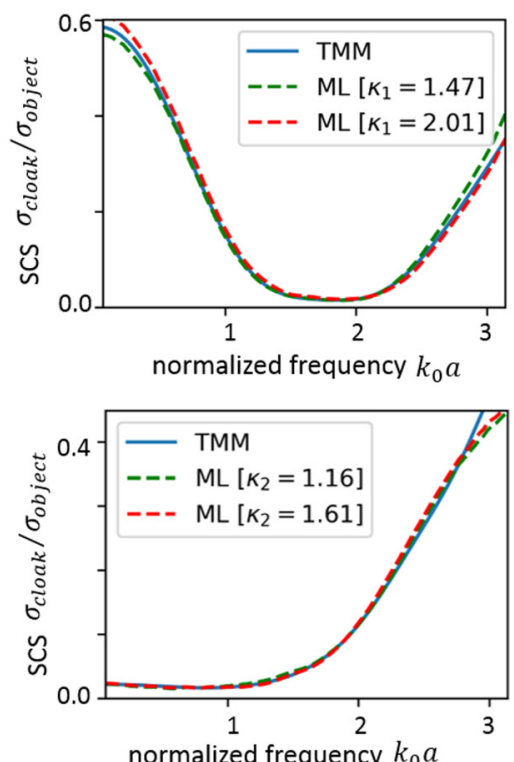

(f)
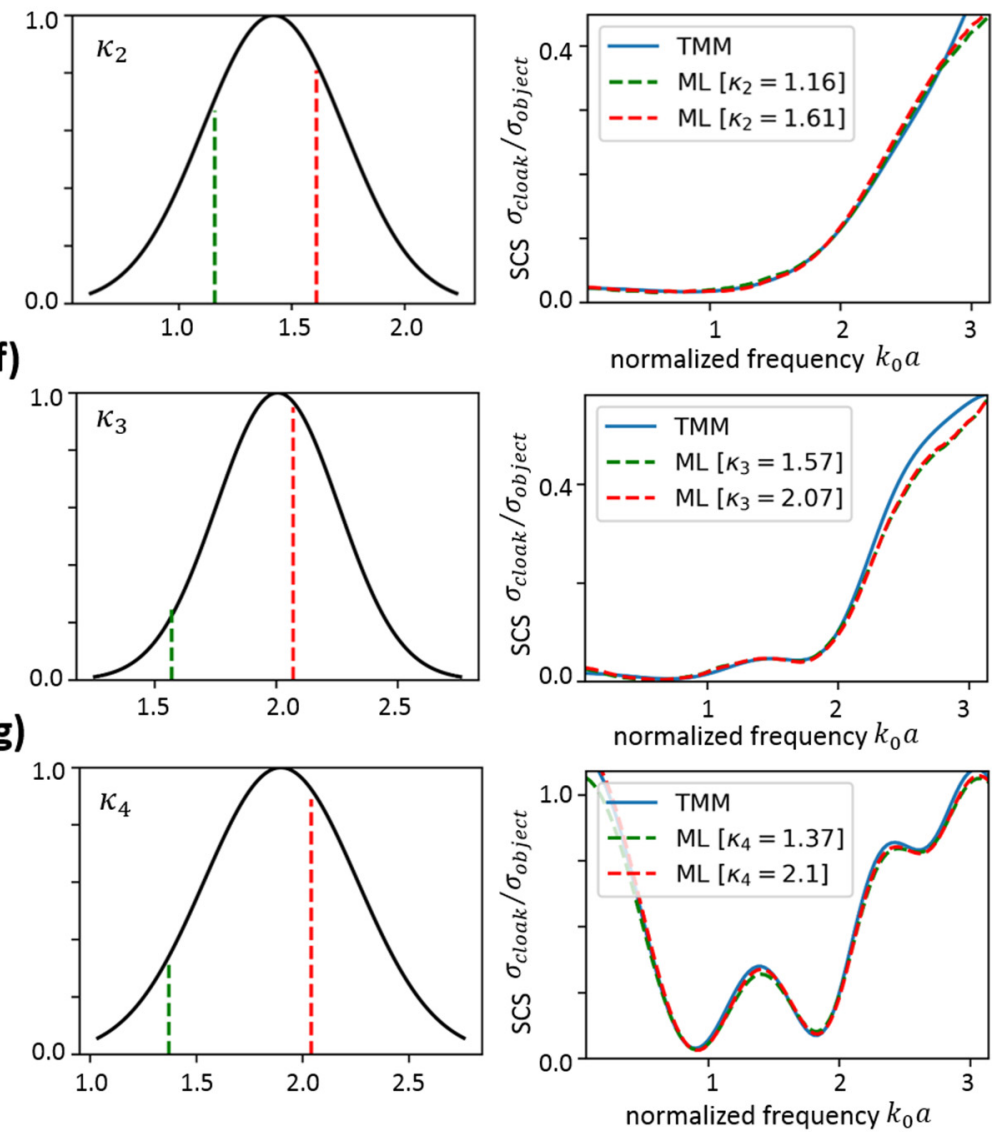

FIG. 5. Probabilistic inverse design of acoustic cloak. (a) Learning curves for the training and validation data sets over training epoch. (b) Classification of testing samples based on maximum standard deviation ( $\pm 2 \%$ and $\pm 4 \%$ deviation from the mean value) in the generated distributions of design parameters. Bulk modulus parameters in design space exhibit large standard deviation. (c) Classification of testing samples based on $\pm 2 \%$ and $\pm 4 \%$ deviation from the mean value in the generated distributions of different combinations of bulk modulus parameters. (d)-(g) Gaussian distributions of bulk moduli showing maximum standard deviation for the representative cases. Comparison of the spectral responses generated from randomly sampled bulk moduli in the corresponding distribution (dashed red and green curves) and reference response (solid blue curve).

model (acting as a decoder) and the reconstruction error is computed. Fourth, the reconstruction error is back propagated through the network to minimize the loss function. We use the normalized parameters for operating frequency and material properties, which can be scaled depending on the choice of the host medium and scatterer. For example, for a $10-\mathrm{cm}$ scatterer immersed in water, the designed cloak perfectly works over a broad spectrum ranging from a few $\mathrm{Hz}$ to $5 \mathrm{kHz}$. To demonstrate this idea, we consider a specific core material to achieve broadband cloaking by tuning the material and geometry of the four-layered core-shell system. However, the approach can be applied to design invisible cloaks for any given core in such systems (see the SM [45] for broadband cloaking of a steel core).

We would like to mention that the cloaking effect can be enhanced by increasing the number of layers for a given frequency range. However, a large number of layers will increase the complexity of the design and will be computationally expensive. Therefore, one should balance the performance and the expenses. In our practice, we choose four layers to demonstrate that our method offers good cloaking effect and provides feasible parameters to realize such a device. The practical implementation of the designed acoustic cloak demands isotropic materials that exhibit the desired 
effective-medium properties. The effective material parameters can be realized by designing disordered [46], extraordinary crystalline [47], and quasicrystalline architectures [48]. Chiral quasicrystalline metamaterials can be designed to obtain the effective parameters of the designed cloak [48].

To summarize, we demonstrate the machine learning driven broadband acoustic cloak with multilayer core-shell configuration. In particular, we develop deterministic and probabilistic deep learning models for inverse design of an acoustic cloak that efficiently solves the inverse design problem. The proposed models utilize the encoder-decoder-like structure to solve the one-many mapping problem and retrieve the design parameters for the given spectral response. The forward network, acting as a decoder, is trained independently and cascaded behind the inverse network either in deterministic or probabilistic design. In the probabilistic design, the design parameters in the form of probabilistic latent variables are obtained by sampling from distributions in the latent space. The distributions of the design parameters are used to reveal the sensitivity of the design parameter on the cloaking functionality and the generation of the desired spectral response with diverse design. The probabilistic network is a highly attractive route to improve the robustness of the cloaking effect against the deviation of the design parameters of the cloak. We envision that our approach can be generically utilized to automate the designing process of complex material systems, showing nonunique solution space, with minimum human intervention.

\section{ACKNOWLEDGMENTS}

The work described here is supported by King Abdullah University of Science and Technology (KAUST) Artificial Intelligence Initiative Fund and KAUST Baseline Research Fund No. BAS/1/1626-01-01.
[1] N. Engheta and R. W. Ziolkowski, Metamaterials: Physics and Engineering Explorations (Wiley, New York, 2006).

[2] J. B. Pendry, D. Schurig, and D. R. Smith, Controlling electromagnetic fields, Science 312, 1780 (2006).

[3] U. Leonhardt, Optical conformal mapping, Science 312, 1777 (2006).

[4] D. Schurig, J. J. Mock, B. J. Justice, S. A. Cummer, J. B. Pendry, A. F. Starr, and D. R. Smith, Metamaterial electromagnetic cloak at microwave frequencies, Science 314, 977 (2006).

[5] B. Edwards, A. Alu, M. G. Silveirinha, and N. Engheta, Experimental Verification of Plasmonic Cloaking at Microwave Frequencies with Metamaterials, Phys. Rev. Lett. 103, 153901 (2009).

[6] A. Alu and N. Engheta, Achieving transparency with plasmonic and metamaterial coatings, Phys. Rev. E 72, 016623 (2005).

[7] A. Alu and N. Engheta, Multifrequency Optical Invisibility Cloak with Layered Plasmonic Shells, Phys. Rev. Lett. 100, 113901 (2008).

[8] P. Y. Chen and A. Alu, Mantle cloaking using thin patterned metasurfaces, Phys. Rev. B 84, 205110 (2011).

[9] Y. Yang, L. Jing, B. Zheng, R. Hao, W. Yin, E. Li, C. M. Soukoulis, and H. Chen, Full-polarization 3D metasurface cloak with preserved amplitude and phase, Adv. Mater. 28, 6866 (2016).

[10] R. El-Ganainy, K. G. Makris, M. Khajavikhan, Z. H. Musslimani, S. Rotter, and D. N. Christodoulides, NonHermitian physics and PT symmetry, Nat. Phys. 14, 11 (2018).

[11] Z. Hayran, R. Herrero, M. Botey, H. Kurt, and K. Staliunas, Invisibility on demand based on a generalized Hilbert transform, Phys. Rev. A 98, 013822 (2018).

[12] N. Landy and D. R. Smith, A full-parameter unidirectional metamaterial cloak for microwaves, Nat. Mater. 12, 25 (2013)

[13] S. Tretyakov, P. Alitalo, O. Luukkonen, and C. Simovski, Broadband Electromagnetic Cloaking of Long Cylindrical Objects, Phys. Rev. Lett. 103, 103905 (2009).

[14] S. A. Cummer and D. Schurig, One path to acoustic cloaking, New J. Phys. 9, 45 (2007).
[15] H. Y. Chen and C. T. Chan, Acoustic cloaking in three dimensions using acoustic metamaterials, Appl. Phys. Lett. 91, 183518 (2007).

[16] S. Zhang, X. Cia, and N. Fang, Broadband Acoustic Cloak for Ultrasound Waves, Phys. Rev. Lett. 106, 024301 (2011).

[17] M. Farhat, S. Guenneau, and S. Enoch, Ultrabroadband Elastic Cloaking in Thin Plates, Phys. Rev. Lett. 103, 024301 (2009).

[18] M. Farhat, S. Guenneau, P. Y. Chen, A. Alu, and K. N. Salama, Scattering Cancellation-Based Cloaking for the MaxwellCutaneous Heat Waves, Phys. Rev. Appl. 11, 044089 (2019).

[19] P. Y. Chen, M. Farhat, S. Guenneau, S. Enoch, and A. Alu, Acoustic scattering cancellation via ultrathin pseudosurface, Appl. Phys. Lett. 99, 191913 (2011).

[20] M. D. Guild, M. R. Haberman, and A. Alu, Plasmonic cloaking and scattering cancelation for electromagnetic and acoustic waves, Wave Motion 48, 468 (2011).

[21] D. Goldberg, Genetic Algorithms in Search, Optimization and Machine Learning (Addison Wesley, Boston, 1989).

[22] H. Gao, Y.-f. Zhu, X.-d. Fan, B. Liang, J. Yang, and J.-C. Cheng, Non-blind acoustic invisibility by dual layers of homogeneous single-negative media, Sci. Rep. 7, 42533 (2017).

[23] Y. LeCun, Y. Bengio, and G. Hinton, Deep learning, Nature 521, 436 (2015).

[24] M. I. Jordan and T. M. Mitchell, Machine learning: Trends, perspectives, and prospects, Science 349, 255 (2015).

[25] Y. Zhang, A. Mesaros, K. Fujita, S. D. Edkins, M. H. Hamidian, K. Ch'ng, H. Eisaki, S. Uchida, J. C. Séamus Davis, E. Khatami, and E.-A. Kim, Machine learning in electronic-quantum-matter imaging experiments, Nature 570, 484 (2019).

[26] M. Närhi, L. Salmela, J. Toivonen, C. Billet, J. M. Dudley, and G. Genty, Machine learning analysis of extreme events in optical fibre modulation instability, Nat Commun. 9, 4923 (2018).

[27] C. Barth and C. Becker, Machine learning classification for field distributions of photonic modes, Commun. Phys. 1, 58 (2018).

[28] L. Pilozzi, F. A. Farrelly, G. Marcucci, and C. Conti, Machine learning inverse problem for topological photonics, Commun. Phys. 1, 57 (2018). 
[29] Z. Liu, D. Zhu, S. Rodrigues, K. Lee, and W. Cai, Generative model for the inverse design of metasurfaces, Nano Lett. 18, 6570 (2018).

[30] S. An, C. Fowler, B. Zheng, M. Y. Shalaginov, H. Tang, H. Li, L. Zhou, J. Ding, A. M. Agarwal, C. Rivero-Baleine, K. A. Richardson, T. Gu, J. Hu, and H. Zhang, A deep learning approach for objective-driven all-dielectric metasurface design, ACS Photonics 6, 3196 (2019).

[31] Y.-T. Luo, P.-Q. Li, D.-T. Li, Y.-G. Peng, Z.-G. Geng, S.-H. Xie, Y. Li, A. Alù, J. Zhu, and X.-F. Zhu, Probability-densitybased deep learning paradigm for the fuzzy design of functional metastructures, Research 2020, 8757403 (2020).

[32] J. Peurifoy, Y. Shen, L. Jing, Y. Yang, F. Cano-Renteria, B. G. DeLacy, J. D. Joannopoulos, M. Tegmark and M. Soljačić, Nanophotonic particle simulation and inverse design using artificial neural networks, Sci. Adv. 4, eaar4206 (2018).

[33] D. Liu, Y. Tan, E. Khoram, and Z. Yu, Training deep neural networks for the inverse design of nanophotonic structures, ACS Photonics 5, 1365 (2018).

[34] S. Molesky, Z. Lin, A. Y. Piggott, W. Jin, J. Vucković, and A. W. Rodriguez, Inverse design in nanophotonics, Nat. Photonics 12, 659 (2018).

[35] W. Ma, F. Cheng, and Y. Liu, Deep-learning enabled ondemand design of chiral metamaterials, ACS Nano 12, 6326 (2018).

[36] D. P. Kingma and M. Welling, Auto-encoding variational Bayes, arXiv:1312.6114.

[37] S. So, J. Mun, and J. Rho, Simultaneous inverse design of materials and parameters of core-shell nanoparticle via deeplearning: Demonstration of dipole resonance engineering, ACS Appl. Mater. Interfaces 11, 24264 (2019).

[38] W. Ma, F. Cheng, Y. Xu, Q. Wen, and Y. Liu, Probabilistic representation and inverse design of metamaterials based on a deep generative model with semi-supervised learning strategy, Adv. Mater. 31, 1901111 (2019).
[39] Q. Zhang, H. Yu, M. Barbiero, B. Wang, and M. Gu, Artificial neural networks enabled by nanophotonics, Light Sci. Appl. 8, 42 (2019).

[40] T. W. Hughes, I. A. Williamson, M. Minkov, and S. Fan, Wave physics as an analog recurrent neural network, Sci. Adv. 5, eaay6946 (2019).

[41] Y. Kiarashinejad, S. Abdollahramezani, and A. Adibi, Deep learning approach based on dimensionality reduction for designing electromagnetic nanostructures, npj Comput. Mater. 6, 12 (2020).

[42] L. Xu, M. Rahmani, Y. Ma, D. A. Smirnova, K. Z. Kamali, F. Deng, Y. K. Chiang, L. Huang, H. Zhang, S. Gould, D. N. Neshev, and A. E. Miroshnichenko, Enhanced light-matter interactions in dielectric nanostructures via machine-learning approach, Adv. Photonics 2, 026003 (2020).

[43] C. Qian, B. Zheng, Y. Shen, L. Jing, E. Li, L. Shen, and H. Chen, Deep-learning-enabled self-adaptive microwave cloak without human intervention, Nat. Photonics 14, 383 (2020).

[44] J. Weng, Y. Ding, C. Hu, X.-F. Zhu, B. Liang, J. Yang, J. Cheng, Meta-neural-network for real-time and passive deep-learningbased object recognition, Nat. Commun. 11, 6309 (2020).

[45] See Supplemental Material at http://link.aps.org/supplemental/ 10.1103/PhysRevResearch.3.013142 for description of design methods, nonconvergence and overfitting issue in the inverse problem, details about optimized hyper parameters, and some additional examples for the designed deterministic and stochastic inverse networks.

[46] Radman, X. Huang, and Y. Xie, Topological optimization for the design of microstructures of isotropic cellular materials, Eng. Optim. 45, 1331 (2013).

[47] R. Lakes, Foam structures with a negative Poisson's ratio, Science 235, 1038 (1987).

[48] Y. Chen, M. Kadic, S. Guenneau, and M. Wegener, Isotropic Chiral Acoustic Phonons in 3D Quasicrystalline Metamaterials, Phys. Rev. Lett. 124, 235502 (2020). 